\title{
Short-range wireless communications and reliability
}

\author{
Ismail Guvenc, Sinan Gezici, Zafer Sahinoglu, and Ulas C. Kozat
}

Even though there is no universally accepted definition, short-range wireless communications typically refers to a wide variety of technologies with communication ranges from a few centimeters to several hundreds of meters. While the last three decades of the wireless industry have been mostly dominated by cellular systems, short-range wireless devices have gradually become a more integrated part of our everyday lives over the last decade. The Wireless World Research Forum (WWRF) envisions that this trend will accelerate in the upcoming years: by the year 2017, it is expected that seven billion people in the world will be using seven trillion wireless devices [1]. The majority of these devices will be short-range wireless devices that interconnect people with each other and their environments.

While the reliability of wireless communication systems has been studied in detail in the past, a comprehensive study of different factors affecting reliability for short-range wireless systems and how they can be handled is not available in the literature, to date. The present book intends to fill this gap by covering important reliability problems for short-range wireless communication systems. The scope of the contributions in the book is mostly within the domain of wireless personal area networks (WPANs) and wireless sensor networks (WSNs), and issues related to wireless local area networks (WLANs) are not specifically treated.

Due to the differences in application scenarios, quality of service (QoS) requirements, signaling models, and different error sources and mitigation approaches, the high-rate and low-rate systems will be addressed in separate parts of the book. For the highrate systems covered in Part I, multiband orthogonal frequency division multiplexing (OFDM) and millimeter wave communication systems will be the main focus owing to their significant potential for achieving high throughputs. On the other hand, Part II of the book will be focusing mostly on ZigBee and pulse-based ultrawideband (UWB) communications owing to their benefits for low-rate, low-power, and low-complexity operation. In addition, a third set of chapters within Part III will be addressing some selected topics related to the reliability of short-range wireless communication systems, where the chapters are written from a broader perspective without specifying a certain technology or standard.

The rest of this chapter is organized as follows. First, in Section 1.1, enabling factors for short-range wireless communications are discussed, and differences from long-range wireless systems are summarized. In addition, a comparison of low-rate and high-rate systems in terms of application scenarios, typical transmitter/receiver 
characteristics, and reliability requirements is provided, and globally available frequency bands for short-range wireless systems are reviewed. In Section 1.2, reliability problems observed at different layers of the protocol stack are defined, and possible solutions to address these are discussed along with references to different chapters in the book. Section 1.3 provides a brief review of certain short-range wireless communications standards, leaving the detailed treatment of more established standards to Chapter 2 and Chapter 6.

\subsection{Short-range wireless communications}

\subsubsection{Enabling factors}

There are three significant factors that play an important role for the widespread use and adoption of short-range wireless communications devices in today's world: (i) advancements in the solid-state devices, (ii) developments in the digital communication and modulation techniques, and (iii) developments in related standardization activities.

Advances in the solid-state technology have been an important factor enabling the widespread use of short-range wireless technologies. First, the mass production of devices became possible, decreasing the production cost per unit device. Second, with the new developments, higher center frequencies have become operational for shortrange devices. This implies access to the previously inaccessible frequency bands such as the $2.4 \mathrm{GHz}, 5 \mathrm{GHz}$, and $60 \mathrm{GHz}$ bands of the industrial, scientific, and medical (ISM) bands that will be discussed in more detail in Section 1.1.4. Using higher center frequencies also enables the use of very small antenna elements, of which multiple may easily be embedded within the same device [2]. Today, circuit miniaturization and small-size antennas make it possible to manufacture extremely small radio frequency integrated circuits (RFICs) on chips that contain all the essential system components. For example, CMOS RFIC-on-chip antennas are available for short-range wireless technologies utilizing central frequencies as high as $60 \mathrm{GHz}$ and having chip sizes of less than $1 \mathrm{~mm}^{2}[3,4]$.

Another enabling factor that has an important role in the success of short-range wireless communication systems is the recent developments in digital modulation techniques and transceiver algorithms. For example, direct sequence spread spectrum (DSSS) technology has been used successfully in systems such as the IEEE 802.15.4 WPANs and the IEEE 802.11 WLANs. Through spreading the frequency content of a transmitted signal, DSSS provides advantages such as interference resilience, low-power spectral density, resistance to jamming, and mitigation of multipath effects [5]. Frequencyhopping spread spectrum (FHSS) is another spread spectrum transmission technology that has commonly been used in short-range wireless devices due to its interference resilience. Because of its important advantages in multipath environments, OFDM has recently been a key technology for achieving higher throughputs in short-range wireless communication systems [6,7]. Advantages of OFDM over other relevant competitive technologies include the following: (i) there is no need for time-domain equalization, and 
much simpler frequency-domain equalization techniques can be utilized efficiently, ${ }^{1}$ (ii) it is robust in frequency selective channels owing to the use of a cyclic prefix (CP), and (iii) multiple-input multiple-output (MIMO) is easily implementable with OFDM due to frequency-flat fading at each tone. Due to such advantages, OFDM has been adopted by recent standards such as ECMA-368 (high-rate UWB PHY and MAC [6]) and ECMA387 (high-rate $60 \mathrm{GHz}$ PHY, MAC, and HDMI PAL [7]). Other recent developments that may impact the future of short-range wireless communications include the advances in MIMO techniques for achieving higher data rates and better reliability [9-13], and cognitive radio methods for more efficient and reliable utilization of the wireless spectrum [14-17].

The critical role of standardization bodies in the widespread use of short-range wireless devices should also be emphasized here. Through standardization, related companies and research organizations actively work towards obtaining a well-defined technical specification for a given wireless technology. This brings with it a high potential for the realizability and interoperability of the technology; a better understanding of the application scenarios, potentials, and limitations is achieved, and a consensus is reached on how to implement it in a good way. Several successful short-range wireless devices that we use in our everyday lives today such as WiFi, Bluetooth headsets, wireless keyboards, and ZigBee devices are all the result of long years of standardization. Probably the most important standardization group working on short-range wireless communication technologies is the IEEE 802.15 Working Group for WPANs. As well as the already standardized short-range wireless technologies discussed before, IEEE 802.15 is also working on the standardization of some recent technologies such as wireless body area networks (WBANs), radio frequency identification (RFID) systems, mesh networks, and visible light communications (VLCs). Other standard bodies related to short-range wireless communications include ISA-100 and ECMA standards. A more detailed discussion on related short-range wireless communication standards will be presented in Section 1.3 as well as in other chapters of the book.

\subsubsection{Short-range versus medium/long-range communications}

While short-range wireless technologies span a wide range of application scenarios, they typically have some common characteristics that are significantly different from medium and long-range wireless technologies, such as WLANs, cellular systems, wireless metropolitan area networks, and satellite communication systems. Some of the common features of short-range wireless devices include low-power operation, communication ranges from several centimeters up to a hundred meters, principally indoor operation, omnidirectional built-in antennas, low-complexity and low-price devices, battery operated transmitter/receiver, and unlicensed operation [18].

Short-range wireless devices typically have very low or no mobility, which implies simple and low-complexity receiver architectures compared, for example, to cellular

${ }^{1}$ Note that frequency domain equalization is also possible for single-carrier frequency domain multiple access (SC-FDMA) systems [8]. 
systems. On the other hand, multihop and cooperative communications may be considered as important operational modes for certain short-range wireless communications scenarios (e.g., as in WSNs). This is primarily due to dense deployment scenarios of wireless sensors that collect local information, aggregate it, and communicate to the intended receiver. Such wireless networks should have very low-power operation for extended network life, and overall power consumption may be decreased by transmitting the packets over multiple shorter distance hops rather than over a direct link with longer transmitter-receiver separation. Due to their critical importance for short-range wireless communication systems, multihop and cooperative communication techniques will be treated in detail in Part III of this book.

The QoS requirements (e.g., packet error rate, data rate, and latency) for short-range wireless systems are also quite different from long-range communication technologies and are closely coupled with the application scenarios. In reference [19], the top 10 design rules for short-range communications, which are different from the design rules of long-range networks, have been listed as follows: communication architecture (both point-to-point and point-to-multipoint communications capability), energy awareness, signaling and traffic channels, scalability and connectivity, medium access control and channel access methods, self organization, service discovery, security and privacy issues, flexible spectrum usage, and software-defined radio design.

\subsubsection{High-rate versus low-rate communications}

It is possible to have different sets of taxonomies and classifications for short-range wireless communication technologies. Among some other possible classifications, they may be classified with respect to their communication ranges, mobility characteristics, network topology, QoS requirements, indoor versus outdoor operation, operating frequency/bandwidth, and data rates. Communication ranges of short-range wireless systems may be on the order of several centimeters (e.g., for near field communications (NFCs)), fractions of a meter (e.g., for WBANs), several meters (e.g., WPANs), or from a few meters up to hundreds of meters (WSNs) [20]. The range of passive RFIDs are on the order of tens of centimeters, while active RFIDs may have ranges as large as a hundred meters. Even though short-range wireless technologies typically operate with no mobility or very low mobility, there may be scenarios in which the mobility may be a concern. For example, body movements in WBANs, or movement of the transmitter and/or the receiver in certain WSN applications may introduce mobility related problems that should be taken into account in receiver design. Centralized network topology or distributed network architectures are two common topologies for short-range wireless communication systems.

Despite the aforementioned classifications and several other possible taxonomies, it is difficult to classify different short-range technologies within different groups. The large diversity of application scenarios and requirements, differences in the air interface, and variations in operational ranges even for the same wireless technology are only a few of the factors preventing well-defined taxonomies. In this book, since it provides a relatively uniform and well-defined classification, we choose to study 
Table 1.1 Example applications for short-range wireless communications.

\begin{tabular}{ll}
\hline Low-rate systems & High-rate systems \\
\hline Tele-control for home and building & Wireless USB \\
Wireless microphones and headphones & Internet access and multimedia services \\
Wireless mouse, keyboard, etc. & Uncompressed high-definition video \\
Remote keyless entry, gate openers, etc. & Patient monitoring in hospitals \\
Wireless bar-code readers & Wireless surveillance cameras \\
Wireless sensor networks & Wireless video conferencing \\
Emergency medical alarms & Wireless ad-hoc communications \\
Wireless billing & Wireless peripheral interfaces \\
\hline
\end{tabular}

short-range wireless communications systems by grouping them into two categories: high-rate systems and low-rate systems.

While a clear-cut separation does not exist, high-rate systems are considered for data rates higher than $10 \mathrm{Mbps}$ (up to several Gbps), and they have communication ranges smaller than $10 \mathrm{~m}$. Example application scenarios for high-rate systems include wireless video streaming, wireless file transfer (e.g., wireless USB), wireless video conferencing, and wireless surveillance cameras. Also, as discussed in reference [1], high-rate technologies considered for short-range wireless communication applications are based on multiband UWB [21] and millimeter wave technologies [22,23], and related wireless standards will be discussed in detail in Chapter 2.

Low-rate systems, on the other hand, are considered for low-power and low-complexity applications that do not have significant data rate requirements. While they do not necessarily have long communication ranges, the maximum ranges of low-rate systems may be significantly larger than those of the high-rate systems. Apart from application related requirements, two important reasons for this are as follows: (i) larger communication ranges mean lower levels of received power, which inherently prevent high data rates, and (ii) high-rate systems require a significantly large bandwidth, which is commonly available at higher central frequencies (e.g., $60 \mathrm{GHz}$ spectrum) that are subject to a larger path-loss. WSNs are probably the most common applications for short-range low-rate wireless communication systems. Two important recent wireless technologies that are suitable for low-rate systems are ZigBee and low-rate UWB, and the wireless standards related to these technologies will be reviewed in detail in Chapter 6. Some examples for short-range wireless communications applications for high-rate and low-rate systems are summarized in Table 1.1, and more detailed discussions of the related applications are left to Chapter 2 and Chapter 6.

The QoS requirements as well as possible techniques and protocols for improving the reliability of low-rate and high-rate systems are considerably different. For example, primarily due to application scenarios and requirements, low-power operation becomes more relevant to WSNs, e.g., for environmental sensing applications, where the sensor nodes should operate with the same battery for extended durations. Power efficient routing techniques and cooperative communication methods may also gain more importance 
in such scenarios. While such techniques may also be applied to certain high-rate communication scenarios, one of the most common applications for high-rate systems is the wireless USB, which by definition is point-to-point, and routing and cooperative communications techniques become irrelevant. Due to multiple-antenna capabilities enabled by high-frequency operation of high-rate systems (e.g., for millimeter wave communications), beamforming techniques and protocols may be very important for certain scenarios in order to minimize the interference and improve reliability.

Signaling models utilized by low-rate and high-rate systems may vary greatly. For example, the high-rate ECMA-368 standard has adopted an MB-OFDM based physical (PHY) layer, which facilitates a simple equalization process in the frequency domain. On the other hand, the low-rate IEEE 802.15.4a standard uses pulse-based signal transmissions. It is an ideal signaling scheme, for example, for low-rate WSN applications, in which low-complexity transmitter/receiver architectures may be designed and highly accurate ranging/positioning is supported. Low-complexity transceiver architectures such as the energy detector and the transmitted-reference schemes become possible with pulse-based signaling, whereas FFT/IFFT operations in OFDM-based transmission may increase the transceiver complexity.

\subsubsection{Review of frequency regulations and available frequency bands}

The choice of the central frequency and communication bandwidth is critical for shortrange wireless communication systems. As discussed earlier, high central frequencies may be preferable in many cases, because they facilitate small form-factors owing to small antenna sizes, and enable access to several license-free frequency bands at high frequencies (typically having fewer interference sources). On the other hand, since signal attenuation is directly proportional to the central frequency, wireless devices employing high central frequencies may not communicate reliably over relatively long distances owing to severe signal attenuation. Based on the application requirements of a certain short-range wireless system, before deciding on an operational center frequency, such trade-offs should be evaluated carefully by system designers.

The frequency bands in which short-range wireless devices may operate are in most cases limited to license-free bands. While certain license-free bands are globally available, there are also some license-free bands that are available in only certain regions of the world. The frequency bands that are globally available for short-range wireless devices are the $13.56 \mathrm{MHz}$ band (typically considered for near-field communications), $40 \mathrm{MHz}$ band, $433 \mathrm{MHz}$ band, $2.4 \mathrm{GHz}$ band, and the $5.8 \mathrm{MHz}$ band [5]. Among these, the $2.4 \mathrm{GHz}$ band is the most popular global license-free band, which is commonly used by WLANs and microwave ovens. Another band that is available and commonly used for short-range communications in Europe, the USA, Canada, Australia, and New Zealand is the $868 \mathrm{MHz} / 915 \mathrm{MHz}$ band.

A part of the spectrum that can be used without a license in most countries is the ISM band [24], which also includes some of the frequency bands discussed above. For example, in the USA, popular ISM bands include the 902-928 MHz, $2.4 \mathrm{GHz}$, and 5.7$5.8 \mathrm{GHz}$ bands. Similar to several other frequency bands for unlicensed transmissions, 
Table 1.2 Review of the ISM/U-NII bands, and the spectrum used for UWB and $60 \mathrm{GHz}$ systems in the USA.

\begin{tabular}{lcll}
\hline ISM bands & Power limit & U-NII 5 GHz bands & Power limit \\
\hline $\mathbf{9 0 2 - 9 2 8 ~ M H z}$ & & WiFi $(802.11 \mathrm{a} / \mathrm{n})$ & \\
Cordless phones & $1 \mathrm{~W}$ & $5.15-5.25 \mathrm{GHz}$ & $200 \mathrm{~mW}$ \\
Microwave ovens & $750 \mathrm{~W}$ & $5.25-5.35 \mathrm{GHz}$ & $1 \mathrm{~W}$ \\
Industrial heaters & $100 \mathrm{~kW}$ & $5.47-5.725 \mathrm{GHz}$ & $1 \mathrm{~W}$ \\
Military radar & $1000 \mathrm{~kW}$ & $5.725-5.825 \mathrm{GHz}$ & $4 \mathrm{~W}$ \\
$\mathbf{2 . 4 - 2 . 4 8 3 5} \mathbf{~ G H z}$ & & $\mathbf{6 0 ~ G H z ~ b a n d}$ & \\
Wi-Fi $(802.11 \mathrm{~b} / \mathrm{g})$ & $1 \mathrm{~W}$ & $57-64 \mathrm{GHz}$ & $0.5 \mathrm{~W}$ \\
Microwave ovens & $900 \mathrm{~W}$ & Ultra-wideband & \\
$\mathbf{5} \mathbf{~ G H z}$ & & $3.1-10.6 \mathrm{GHz}$ & $-41.3 \mathrm{dBm} / \mathrm{Mhz}$ \\
$5.725-5.825 \mathrm{GHz}$ & & & \\
Wi-Fi $(802.11 \mathrm{a} / \mathrm{n})$ & $4 \mathrm{~W}$ & & \\
\hline
\end{tabular}

the ISM bands are defined under the Part 15 rules of the Federal Communications Commission (FCC). Until 1985, the industrial, scientific, and medical (ISM) bands were not allowed to be used for radio communications in the USA. Together with the FCC Part 15.247 rules in 1985, the ISM bands have been opened for use by WLANs and mobile communications [24]. The Unlicensed National Information Infrastructure (UNII) bands introduced by Part 15.401 to Part 15.407 of the FCC in 1997 added additional license-free frequency bands in the $5 \mathrm{GHz}$ range.

In 2002, the FCC released the Subpart-F of its Part 15 rules, which defines the scope and operation of UWB devices (including communications, imaging systems, and ground-penetrating radar) under Part 15.501 to Part 15.525. Based on this new ruling, UWB devices can transmit at power levels up to $-41.3 \mathrm{dBm} / \mathrm{MHz}$ in the frequency spectrum between $3.1 \mathrm{GHz}$ and $10.6 \mathrm{GHz}$. This opens up a large amount of spectrum available for use by short-range UWB wireless devices. Another large spectrum that can be utilized by short-range wireless devices is defined by the Part 15.255 rules of the FCC, which allow transmission powers up to $500 \mathrm{~mW}$ within the frequency range 57$63 \mathrm{GHz}$. This spectrum is commonly referred to as the millimeter wave or the $60 \mathrm{GHz}$ spectrum, and is another popular band for future short-range high-rate communication systems. The frequency bands and transmit power limits for the ISM/U-NII bands, UWB, and $60 \mathrm{GHz}$ systems in the USA are summarized in Table 1.2. More details on the unlicensed frequency bands of the FCC can be found in reference [25], while further discussions about the sub-GHz frequency bands around the world for short-range wireless communication systems can be found in reference [5].

\section{$1.2 \quad$ Definition of reliability}

The focus of the current book is on reliability aspects of short-range wireless communication systems. Ultimately, reliability should be defined by the application 
itself. For some applications (e.g., data transfer), reliability is about data integrity and all the information sent by the transmitter must be accurately received at the receiver. For other applications such as audio and video, it is less about data integrity and more about tolerable distortion at the application layer which is a convoluted function of error rates, error burstiness, delay, error concealment techniques, etc. Traditionally, each layer of the communication stack addresses reliability at different timescales to fix errors that are not correctable, observable, or too costly to correct at the lower layers. In wireless systems, however, independent decisions at each layer often lead to an unreliable or inefficient communication environment. Therefore, some degree of cross-layer coordination/optimization has been proposed by numerous research papers and adopted in some systems (especially between the PHY and medium access control (MAC) layers). In different chapters, examples of such cross-layer optimization and coordination will be treated in their special contexts. In the rest of this section, we briefly overview how reliability is impacted by the decisions at different layers of the communication stack and discuss error sources from the perspective of each layer.

\subsubsection{Reliability at the PHY layer}

The PHY layer in a digital communication system is responsible for bit-level transmission/reception of signals between the nodes. It has to ensure that the transmitted bits are reliably reconstructed at an intended receiver. In order to understand better the basic principles of digital transmission/reception and related error sources involved at the PHY layer, a simple example of a transmitter/receiver architecture is illustrated in Figure 1.1. The chapters that will be addressing different aspects of reliability are illustrated in the figure. At the transmitter, data to be communicated to a target receiver is in the form of bits, composed of 0's and 1's. These bits are mapped onto signal waveforms after a modulation/coding stage. Through an RF oscillator, the transmit waveform is up-converted to the desired central frequency, amplified, and transmitted through the antenna. Before the transmit waveform arrives at the receiver, it propagates through the wireless channel, which may distort the transmitted signal in different ways as illustrated in Figure 1.1. Once the signal arrives at the receiver, it passes through the low-noise amplifier (LNA) and down-conversion stages, and gets demodulated/decoded to obtain the received bits. The transmitter structure of short-range wireless devices defined in specific standards will be discussed in more detail in Chapter 2 and Chapter 6.

Some of the important metrics that characterize reliability at the physical layer include the signal to interference plus noise ratio (SINR), bit error rate (BER), symbol error rate (SER), packet error rate (PER), and outage probability. Certain issues related to the reliability and relevant error sources at the PHY layer may also be explained through the help of basic channel capacity formulations. In reference [26], a reliable communication is defined as having an arbitrarily small error probability $P_{\mathrm{b}}$, and the maximum data rate at which reliable communication is possible is defined as the capacity $C$ of the channel. Achievable capacity for reliable communications may simply be written for additive 


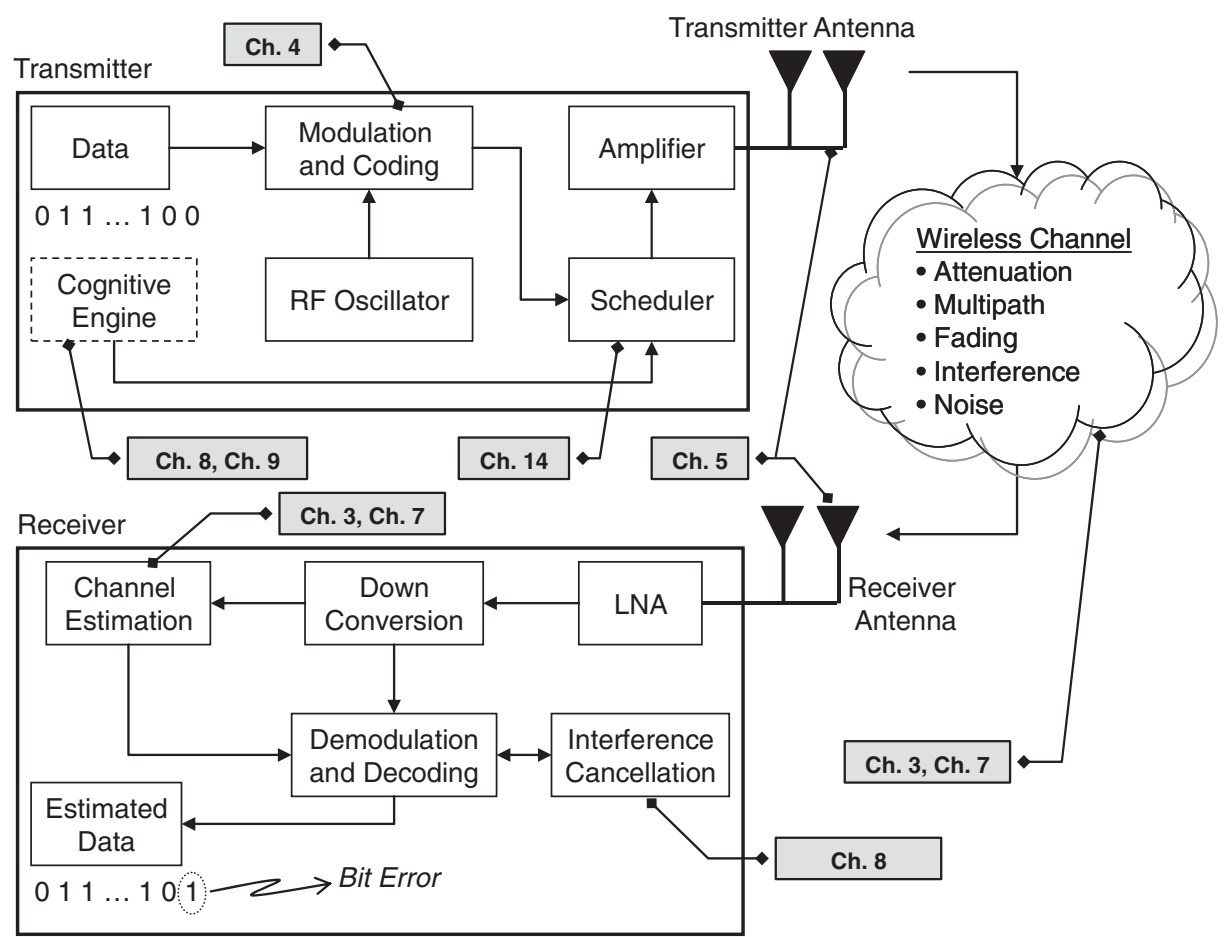

Figure 1.1 An example block diagram of a wireless transmitter/receiver and related error sources.

white Gaussian noise (AWGN) channels as ${ }^{2}$

$$
C_{\text {awgn }}=B \log \left(1+\frac{P_{\text {rec }}}{\sigma_{\mathrm{I}}^{2}+\sigma_{\mathrm{n}}^{2}}\right),
$$

where $B$ is the communication bandwidth, $P_{\text {rec }}$ is the received power of the signal, $\sigma_{\mathrm{I}}^{2}$ captures the variance of different error/interference terms (which are assumed to be white Gaussian processes independent from the noise term), $\sigma_{\mathrm{n}}^{2}=B N_{0}$ is the noise variance, $N_{0}$ is the noise spectral density, and $\frac{P_{\text {rec }}}{\sigma_{\mathrm{I}}^{2}+\sigma_{\mathrm{n}}^{2}}$ is referred to as the SINR. Note that while the interference is assumed to be Gaussian in (1.1), this holds only with a sufficiently large number of interferers, which may not always be the case for short-range systems. As the channel capacity in (1.1) increases, reliable communications become possible at higher data rates. In order to increase the capacity, the bandwidth $B$ can be increased (e.g., through scheduling algorithms), average interference power $\left(\sigma_{I}^{2}\right)$ can be decreased (e.g., through interference cancellation techniques), or the received power $P_{\text {rec }}$ can be increased (e.g., through power control algorithms).

In the rest of this subsection, Figure 1.1 and equation (1.1) will be used to discuss the major error sources that may impact the reliability at the PHY layer. Possible techniques that may be used in order to mitigate the undesired effects of these error sources will

2 This may be easily extended to include different types of MIMO techniques, impact of multipath channels, cooperative communications, etc. [26]. 
also be explained, along with referrals to the related chapters in the book for a more complete treatment.

\subsubsection{Attenuation}

The received power $P_{\text {rec }}$ in (1.1) should be sufficiently larger than the combination of noise and interference powers for the reliable detection of received bits. Due to path loss, the received power is less than the transmitted power. In free space, the Friis formula relates the transmitted and received powers as follows:

$$
P_{\text {rec }}=P_{\mathrm{t}} \frac{\lambda^{2} G_{\mathrm{t}} G_{\mathrm{r}}}{(4 \pi d)^{2}}
$$

where $P_{\mathrm{t}}$ denotes the transmit power, $\lambda=c / f_{\mathrm{c}}$ is the wavelength, $c$ is the speed of light, $f_{\mathrm{c}}$ is the central frequency, and $G_{\mathrm{t}}$ and $G_{\mathrm{r}}$ are the antenna gains at the transmitter and the receiver, respectively. Since free-space propagation may not describe most environments accurately, a better approach is to use the empirical path loss formula

$$
P_{\text {rec }}=P_{\mathrm{t}} P_{\mathrm{o}}\left(\frac{d_{\mathrm{o}}}{d}\right)^{\alpha} \chi_{\mathrm{sh}},
$$

where $P_{\mathrm{o}}$ is the measured path loss at a reference distance $d_{\mathrm{o}}$ (typically well approximated by $(4 \pi / \lambda)^{2}$ for $d_{\mathrm{o}}=1$ [27]) and $\alpha$ is the path loss exponent. The path loss is also subject to shadowing effect due to several obstacles between the transmitter and receiver, that is captured by the multiplicative term $\chi_{\mathrm{sh}}$ in (1.3). The shadowing is typically modeled using a log-normal random variable, where $10 \log _{10} \chi_{\mathrm{sh}} \sim \mathcal{N}\left(0, \sigma_{\mathrm{s}}^{2}\right)$, with $\sigma_{\mathrm{s}}^{2}$ denoting the variance of $\chi_{\mathrm{sh}}$ in the logarithmic scale.

It is obvious from both (1.2) and (1.3) that the path loss is directly proportional to the central frequency. Therefore, wireless communication systems operating at higher central frequencies (e.g., operating in the millimeter wave spectrum) may have significantly shorter communication distances than wireless devices operating at lower central frequencies. Similarly, the path loss is also directly proportional to the propagation distance. Therefore, the receivers that are closer to the transmitter will have larger received powers while far-away receivers will have lower received powers, implying lower reliability based on (1.1). A method to tackle this problem is to use adaptive modulation and coding (AMC) schemes, which adaptively select the modulation/coding scheme based on the received signal quality. When the received signal quality is good, higher order modulation schemes such as 64-QAM can be utilized to achieve higher data rates. If the receiver moves away from the transmitter, the received signal quality degrades and the receiver is no longer able reliably to demodulate the received bits with 64-QAM. Hence, a lower order modulation such as binary phase shift keying (BPSK) can be used, where the distance between the constellation points is larger, enabling reliable demodulation of the bits at the expense of lower data rates. The AMC schemes for high-rate systems will be discussed in more detail in Chapter 4.

Another possible way to improve the system performance in the presence of variations in the received signal power is to employ power control techniques. For users far away from the transmitter, a larger transmit power may be used to ensure sufficiently large 
received powers at the receiver. The power may also be focused along a certain beam direction using beamforming techniques, which will be discussed in detail in Chapter 5 . In order to improve network lifetime, energy saving approaches at the MAC layer are also commonly considered, which will be discussed in detail in Chapter 10 .

\subsubsection{Multipath propagation}

Apart from path-loss and shadowing, the received signal power is also subject to variations (selectivity) in time, frequency, and space. These three characteristics of the channel have critical impacts on receiver design and the reliability of communications. In particular, the channel should accurately be estimated for reliable detection of transmitted symbols. Channel models for short-range wireless systems will be reviewed in Chapter 3 and Chapter 7 along with the related channel estimation techniques for high-rate and low-rate systems, respectively.

While accurate channel estimation is critical for reliable communications, different multiple antenna techniques may also be used in order to improve reliability by utilizing the selectivity of the wireless channel in time, frequency, and space. The data rate of a multiple antenna system may be improved using spatial multiplexing techniques, where the achievable capacity scales with $\min \left\{N_{\text {tx }}, N_{\text {rx }}\right\}$, with $N_{\text {tx }}$ and $N_{\text {rx }}$ denoting the number of transmitter and receiver antennas, respectively [26]. On the other hand, multiple antennas may also be used to increase the reliability through diversity techniques. For example, through transmit diversity techniques, identical information is transmitted over multiple antennas, each of which goes through independently fading channels. Receiver diversity techniques, on the other hand, utilize multiple receiver antennas, which again observe independently faded replicas of the transmitted signal. Through intelligent combining of the multiple and independently faded replicas of the transmitted signal at the receiver, a more reliable demodulation of the received signal can be obtained. This tradeoff between the capacity and the reliability of a wireless system with multiple antennas is commonly referred to as the diversity-multiplexing tradeoff [28]. Several variations of MIMO and smart antenna techniques for short-range high-rate wireless communications will be discussed in detail in Chapter 5.

\subsubsection{Interference sources}

Interference factors such as multiuser interference and narrowband interference may make the $\sigma_{\mathrm{I}}^{2}$ term in (1.1) larger, and hence degrade the SINR and the reliability of the received signals. Short-range wireless communication systems typically have to coexist with various technologies utilizing the frequency bands summarized in Table 1.2. Therefore, they may receive interference from (and cause interference to) other wireless technologies such as the WLANs that operate within the unlicensed bands.

There may be several approaches for improving the reliability in the presence of interference from other wireless devices. For example, cognitive radio techniques can be utilized to sense the interference sources and try to avoid them [14]. Along these lines, in Chapter 9, spectrum sensing techniques and some related experimental results for lowrate systems will be presented. In some cases, however, it may not be possible to avoid interference, necessitating the use of interference cancellation methods. Cancellation of 
multiuser and narrowband interference for short-range wireless communication systems will be discussed in detail in Chapter 8 .

\subsubsection{Reliability at the MAC layer}

At the MAC layer, reliability is traditionally defined from the data integrity point of view and packets erroneously received from the physical layer are dropped. Thus, a critical metric at this layer is the packet drop rate (PDR) and at least for point-to-point unicast transmissions MAC layer designs aim at marginalizing the packet drops due to link/channel errors. Collision-free channel access and coded or uncoded packet retransmissions are the main mechanisms employed at this layer to improve the PDR. On the other hand, many wireless MAC designs do not attempt to fix packet errors for point-to-multipoint (i.e., multicast/broadcast) wireless transmissions. Instead, low-rate transmissions for such sessions are used for increased reliability in terms of PERs. More recently, a combination of erasure coding at the MAC layer and rate control at the PHY layer has been proposed as a promising technique for various multicast/broadcast scenarios [52]. Since in short-range radio there are fewer receivers (with possibly more correlations in their channel conditions) to be served in comparison to broadcasting in terrestrial or satellite-type services, feedback might be a plausible option even for multicast/broadcast-type services. Cross-layer optimization and cooperative communications have been other recent areas of focus that require tight coordination between the MAC layer and other layers including physical and routing layers to improve reliability in multiple access and multicast channels. Chapters 11 to 14 provide an interesting spectrum of research results with an in-depth treatment of particularly important ones.

Limiting the reliability to data integrity and/or packet drop rates at the MAC layer is quite a narrow view once the requirements of several short-range radio applications are considered. In one set of applications such as multimedia and interactive applications, forcing low packet error rates indiscriminately might induce excessive delays due to retransmissions rendering the received packets useless at the application layer. Delay and jitter are directly impacted by the MAC layer decisions. The scheduling problem might be quite hard even under fixed channel conditions and error-prone wireless channels coupled with such scheduling decisions lead to an even greater challenge. In this respect, many efforts are dedicated to cross-layer optimization both in single-hop and multihop wireless networks [51]. Some of those techniques are presented in Chapter 14. Another critical reliability measure that mainly the MAC layer decisions govern is the network or device lifetime, which is of paramount importance for battery-powered environments. Several MAC design choices and the design tradeoffs for energy efficiency are discussed in more detail in Chapter 10.

\subsubsection{Reliability at the routing layer}

At the routing layer, reliability traditionally targets end-to-end connectivity and maintenance of sufficiently high-quality communication paths under dynamic network conditions. Network conditions might vary as a result of node or link failures, mobility, 
changes in wireless channel quality, changes in traffic demand, etc. Depending on the particular scenario, few of these network dynamics become the dominant characteristics and routing protocols can be customized accordingly with various notions of reliability. For instance, many works on routing in wireless networks in the context of mobile adhoc networks (MANET) have mainly focused on developing protocols that can work in high-mobility scenarios. With links forming and tearing up quite fast, route discovery and packet losses due to lack of connectivity are the main reliability issues that have been investigated. Therefore, routing protocols in MANET scenarios have been evaluated principally with respect to their overhead versus packet delivery ratios, mainly under deterministic coverage models [54,55].

When wireless nodes are quasi-stationary or stationary, other aspects, such as losses due to unreliable wireless channel conditions and to congestion, network stability, delay, and network capacity, surface as critical objectives moving away from connectivityoriented routing layer reliability. In the context of wireless mesh networks and sensor networks, these different angles of reliability have been tackled to a degree. Some of the notable developments to increase the reliability of the routing layer range from the devising of new routing metrics [56] to developing better protocols that utilize techniques such as multipath diversity, opportunistic routing, back-pressure algorithms, cooperative communications, erasure and network coding. Many of these methods take full advantage of the broadcast medium and cross-layer optimization with the PHY and MAC layers being important aspects. Some of these techniques are treated in Chapters 11 to 13. In particular, Chapter 11 investigates cooperative communication techniques with emphasis on virtual beamforming and rateless coding. Authors construct a building block network and protocols over a simpler relay channel model. Authors also investigate how to perform routing and resource allocation in large networks based on these building blocks. Chapter 12, in contrast, focuses on the relay selection problem in block fading channels to boost the communication reliability against channel outages. Chapter 13 focuses on power-limited low SNR wideband communication scenarios. End-to-end scaling performance limits of various relaying and multihop routing algorithms and architectures in large-scale distributed wireless networks are investigated in depth. The analysis formalizes multihop communication as another form of diversity.

Going beyond communications, in some more specialized areas such as in certain sensor network applications, routing also facilitates and maintains high-quality (distributed) computation, generates data compression opportunities, and/or forms a network-wide storage. Routing plays an important role also in terms of network and node lifetimes, since it ultimately determines the load of each relay node in the system [53].

A summary of how different error sources are handled in MAC and routing layers and respective chapters in which they are handled is illustrated in Figure 1.2.

\subsection{Review of related wireless standards}

In order to provide harmonization of various short-range wireless systems, standardization efforts are in progress. The main body that organizes standardization activities is the 


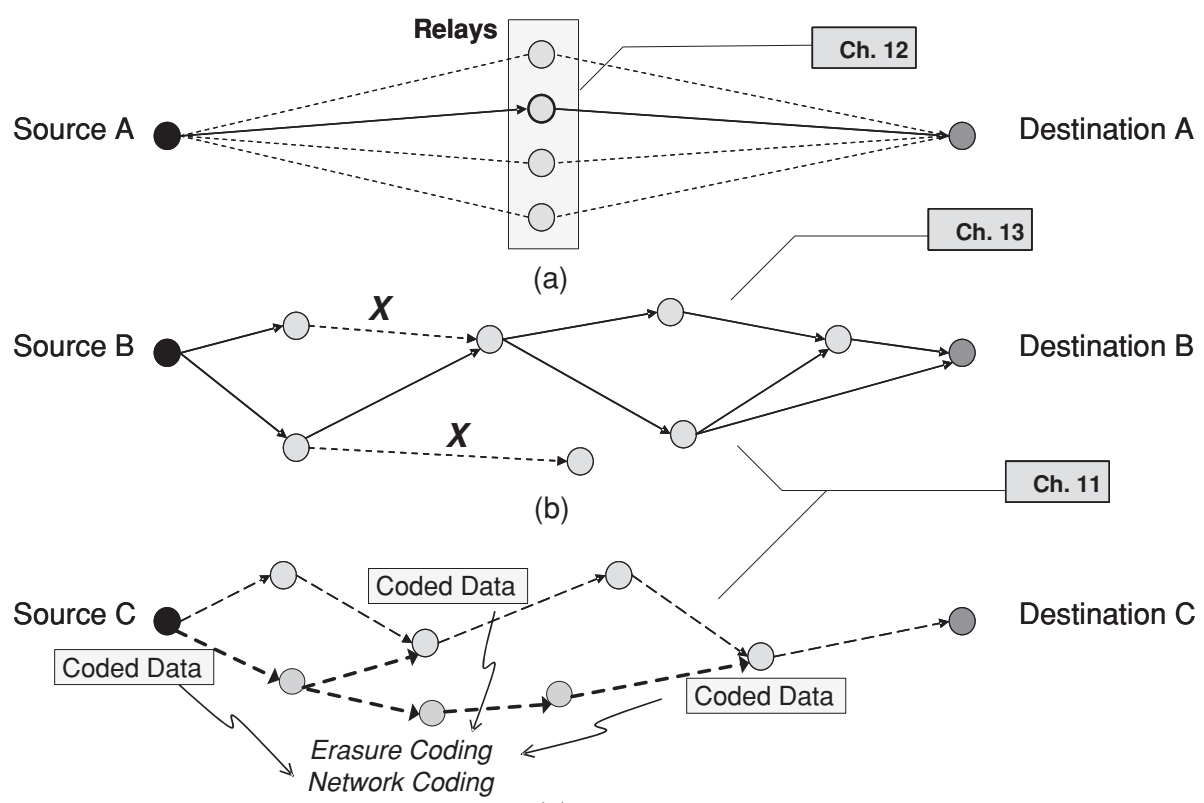

(c)

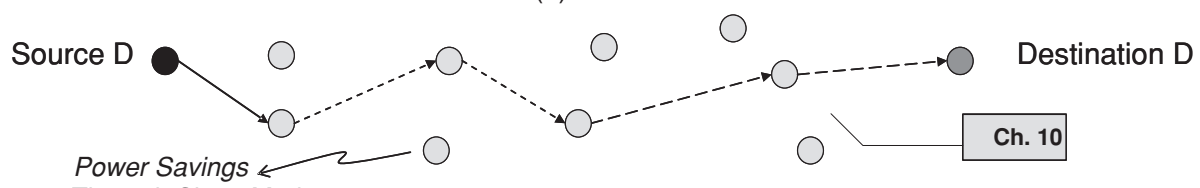

(d)

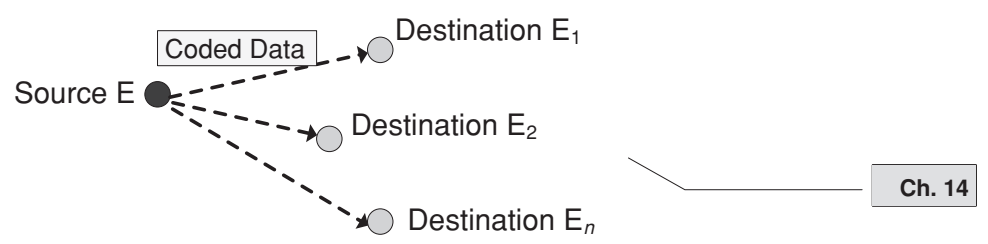

(e)

Figure 1.2 Different techniques for achieving reliability at the MAC/routing layers; (a) relay selection, (b) cooperative communications, (c) error/erasure correction codes, (d) routing and power saving mechanisms, (e) coded opportunistic scheduling.

IEEE, which formed the IEEE 802.15 Working Group for WPAN for the development of consensus standards for short-range wireless networks [29].

In Table 1.3, the task groups (TGs) under the IEEE 802.15 Working Group are listed. TG1 focused on Bluetooth devices and provided a standard for the initial versions of Bluetooth. However, the main activities on the standardization of Bluetooth have been undertaken by the Bluetooth Special Interest Group (SIG) [30], and the later versions of Bluetooth have not been ratified as IEEE standards (see Section 1.3.1). TG2 was formed to develop coexistence mechanisms for the coexistence of WPANs and WLANs, 
Table 1.3 Task groups (TGs) in IEEE 802.15 Working Group for WPAN [29].

\begin{tabular}{lll}
\hline Name & Description & IEEE standard \\
\hline TG1 & Bluetooth & IEEE 802.15.1-2002 \\
TG2 & Coexistence of WPAN (802.15) and WLAN (802.11) & IEEE 802.15.2-2003 \\
TG3 & High-rate WPAN & IEEE 802.15.3-2003 \\
TG3a & High-rate alternative PHY & None \\
TG3b & MAC amendment to IEEE 802.15.3-2003 & IEEE 802.15.3b-2005 \\
TG3c & Millimeter wave alternative PHY & IEEE 802.15.3c-2009 \\
TG4 & Low-rate WPAN & IEEE 802.15.4-2003 \\
TG4a & Low-rate alternative PHY with UWB and CSS & IEEE 802.15.4a-2007 \\
TG4b & Enhancements to IEEE 802.15.4-2003 & IEEE 802.15.4-2006 \\
TG4c & PHY amendment to IEEE 802.15.4-2006 and IEEE 802.15.4a-2007 & IEEE 802.15.4c-2009 \\
TG4d & Amendment to IEEE 802.15.4-2006 & IEEE 802.15.4d-2009 \\
TG4e & MAC amendment to IEEE 802.15.4-2006 & In progress \\
TG4f & Active RFID system & In progress \\
TG4g & Smart utility networks & In progress \\
TG5 & Mesh networking & IEEE 802.15.5-2009 \\
TG6 & Body area networks (BANs) & In progress \\
TG7 & Visible Light Communications (VLC) & In progress \\
\hline
\end{tabular}

and published the IEEE 802.15.2-2003 standard that focuses on the coexistence of Bluetooth devices based on the IEEE 802.15.1-2002 standard and WLANs based on the IEEE 802.11b-1999 standard [31]. Since the ongoing efforts on new WPAN and WLAN standards affect the coexistence mechanisms between the networks, TG2 decided to stop its activities and is now in hibernation until further notice [32].

TG3 is the high-rate task group for WPANs and it aims for high-rate (above $20 \mathrm{Mbps}$ ), low-power and low-cost solutions for portable consumer digital imaging and multimedia applications [33]. After TG3 published the IEEE 802.15.3-2003 standard for high-rate WPANs, a new task group TG3b provided an amendment, IEEE 802.15.3b-2005, to the standard for MAC layer enhancements. IEEE 802.15.3-2003 and IEEE 802.15.3b-2005 are studied in Section 1.3.2.2 in more detail. In 2005, TG3c was formed to provide an amendment to the IEEE 802.15.3-2003 standard for an alternative PHY based on the millimeter wave technology. The activities of TG3c and the millimeter wave technology are discussed in Section 2.4 of Chapter 2. Another attempt to provide an alternative PHY was taken by TG3a, which aimed for a PHY based on UWB technology. However, TG3a was not able to choose between the two PHY proposals and had to stop its activities without a standard. High-rate WPANs based on the UWB technology were standardized by ECMA [34,35]; this is discussed in detail in Section 2.2 of Chapter 2.

TG4 is the low-rate task group for WPANs, and published the IEEE 802.15.4-2003 standard. The standard aims to provide low-cost, low-rate, and ubiquitous communication between wireless devices. Low-rate WPANs and related standards are discussed in Chapter 6. The activities of TG5, TG6, and TG7 are studied within this chapter in Sections 1.3.2, 1.3.3, and 1.3.4, respectively.

In addition to the IEEE standards mentioned above, there are also standards on short-range wireless systems developed by other standardization bodies, such as ECMA 
Table 1.4 Different classes for Bluetooth devices.

\begin{tabular}{lcc}
\hline Class & Maximum power $(\mathrm{mW})$ & Range $(\mathrm{m})$ \\
\hline Class 1 & 100 & 100 \\
Class 2 & 2.5 & 10 \\
Class 3 & 1 & 1 \\
\hline
\end{tabular}

International [36] and ISA [37]. Also, a large number of proprietary systems are available in the market. A brief discussion on the ISA SP-100 standard for process control and monitoring is provided in Section 1.3.5, while a detailed review of ECMA standards for UWB and millimeter wave communication systems will be provided in Chapter 2 .

\subsubsection{Bluetooth}

Bluetooth is a WPAN standard for exchanging data over short distances. It is employed in many personal devices today, such as mobile phones and laptops. Bluetooth was originally developed by Ericsson in 1994. Then, the Bluetooth SIG was formed in 1998 with five companies, and the Bluetooth 1.0 specification was released in 1999 [30]. The next versions, Bluetooth 1.1 and Bluetooth 1.2, were also IEEE standards, namely, IEEE Standard 802.15.1-2002 and IEEE Standard 802.15.1-2005, respectively [38,39]. The first versions of Bluetooth employ Gaussian frequency shift keying (GFSK) and provide data rates up to $721 \mathrm{kbps}$.

The second versions of Bluetooth, Bluetooth 2.0 and Bluetooth 2.1, provide an enhanced data rate (EDR) feature and can reach data rates of 2.1 Mbps. EDR uses GFSK for the packet header and the access code ${ }^{3}$ and $\pi / 4$ differential quaternary phase-shift keying ( $\pi / 4$-DQPSK) or eight-phase differential phase-shift keying (8-DPSK) for the payload [40]. The use of PSK in the payload provides the increase in the data rate.

The Bluetooth devices operate in the $2.4 \mathrm{GHz}$ unlicensed ISM band, that is from $2.4 \mathrm{GHz}$ to $2.4835 \mathrm{GHz}$. A Bluetooth system uses 79 channels in this band, that are indexed as $2402+k \mathrm{MHz}$ for $k=0,1, \ldots, 78$. Since each channel is $1 \mathrm{MHz}$, the operating frequency range is given by [2.4015, 2.4805] GHz. Each channel is divided into time slots for time-division duplexing (TDD), and FHSS is used to combat the adverse effects of wireless channels, such as fading and interference. Frequency hoppings can take place between 79 or fewer channels and a standard hop rate of $1600 \mathrm{hop} / \mathrm{s}$ is employed [39]. In addition, the Bluetooth standard provides three classes with different power-range tradeoffs as shown in Table 1.4.

The Bluetooth system supports point-to-point and point-to-multipoint connections. Two or more devices with the same PHY form an ad-hoc network (piconet). One device is designated as the master, and up to seven other devices can join the piconet as slaves. All the devices in a piconet are synchronized to a common clock reference and frequency hop pattern, which is determined by the master device [40,41].

\footnotetext{
3 The access code is used by the receiver to recognize incoming transmissions.
} 


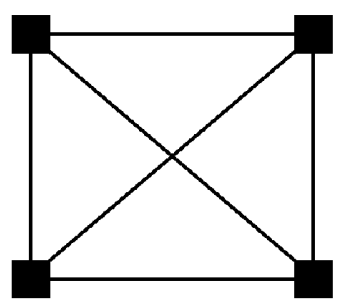

(a)

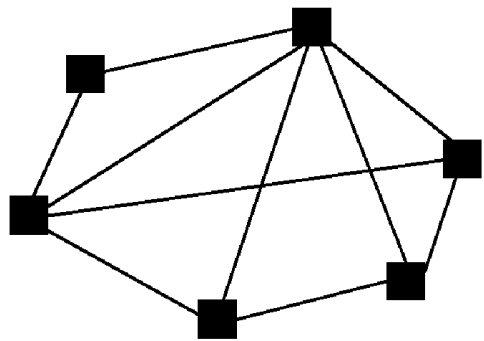

(b)

Figure 1.3 (a) Full mesh topology (b) partial mesh topology.

Recently, Bluetooth 3.0 specification has been announced by the Bluetooth SIG, which integrates the previous Bluetooth technology with 802.11. Bluetooth 3.0 has the Alternate MAC/PHY (AMP) feature, which facilitates the use of alternative MAC and PHY layers to transfer Bluetooth profile data. By this method, transmission of large amounts of data can be performed much faster than the previous versions of Bluetooth. However, the conventional Bluetooth techniques are still employed for device discovery, initial connection, and profile configuration, which provide an overall system with low power consumption [42].

\subsubsection{IEEE 802.15.5 (mesh networking)}

The IEEE 802.15.5 standard specifies the necessary mechanisms that must be present in the PHY and MAC layers of WPANs to facilitate wireless mesh networking (WMN) $[43,44]$. WMN enables dynamic self-organization and self-configuration, meaning that the nodes in the network can automatically form an ad-hoc network and maintain mesh connectivity [45]. A WMN is a fully connected network if each node is connected directly to each of the other nodes, and is also called the full mesh topology. However, in the partial mesh topology, some nodes are connected to all the others, but some are connected only to those other nodes with which they exchange the most data [44]. In Figure 1.3, examples of full and partial mesh topologies are illustrated. The main advantages of the full mesh topology are improved reliability and efficiency. However, these advantages are accompanied by high cost, since a large number of links are needed. Specifically, for a fully connected network with $N$ nodes, $N(N-1) / 2$ links need to be formed.

The IEEE 802.15.5 standard aims to optimize wireless mesh topologies for WPANs in order to provide the following features [43]:

- extension of network coverage without increasing the transmit power or the receiver sensitivity;

- enhanced reliability via route redundancy;

- easier network configuration;

- improved battery life. 


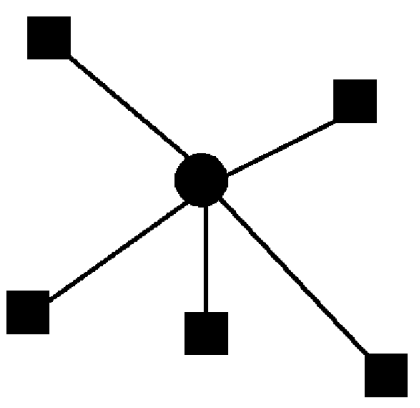

(a)

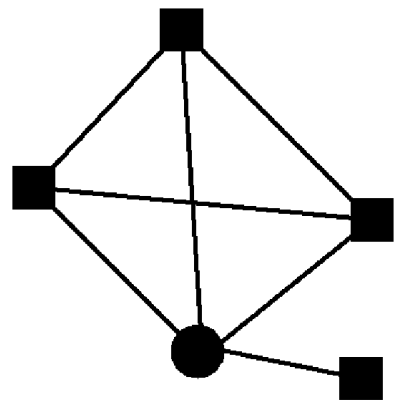

(b)

Figure 1.4 Network topologies in the IEEE 802.15.4-2006 standard, where circles represent the PAN coordinators: (a) star topology; (b) peer-to-peer topology.

The standard describes WMN for low-rate WPANs and high-rate WPANs based on related IEEE standards, as studied next.

\subsubsection{Low-rate WPAN mesh}

The IEEE 802.15.5 standard [43] provides an architectural framework to facilitate interoperable, stable, and scalable wireless mesh topologies for low-rate WPANs based on the IEEE 802.15.4-2006 standard [46]. ${ }^{4}$ Originally, IEEE 802.15.4-2006 supported the star topology and the peer-to-peer topology, as shown in Figure 1.4. In the star topology, the devices are connected to a single central controller, called the personal area network (PAN) coordinator. However, in the peer-to-peer topology, a device can form a connection with any other device as long as they are in range of each other. Although the peer-to-peer topology allows mesh networking to be realized in WPANs, the IEEE 802.15.4-2006 standard does not specify how mesh networking should be implemented.

The IEEE 802.15.5 standard describes a standard way of performing mesh networking over IEEE 802.15.4-2006, and provides supports for the following features [43]:

- unicast, multicast, and reliable broadcast mesh data forwarding;

- synchronous and asynchronous power saving for mesh devices;

- trace route function;

- portability of end devices.

Low-rate WPAN mesh networks have various applications, such as automation and control, safety, security, environment monitoring, and automatic meter reading $[43,47]$. As a specific example, it is stated in reference [43] that, via WPAN mesh networks, wireless light switches in a commercial building (e.g., in a department store) can control the lights of an entire floor, with the ability to group lights in different ways in a dynamic manner and turn them on/off with a single push of a button.

\footnotetext{
4 The IEEE 802.15.4-2006 standard is studied in detail in Section 6.2 of Chapter 6.
} 
Table 1.5 Different modulation and coding types in IEEE 802.15.3, where TCM refers to trellis coded modulation [48].

\begin{tabular}{lll}
\hline Modulation & Coding & Data rate (Mbps) \\
\hline QPSK & 8-state TCM & 11 \\
DQPSK & None & 22 \\
16-QAM & 8-state TCM & 33 \\
32-QAM & 8-state TCM & 44 \\
64-QAM & 8-state TCM & 55 \\
\hline
\end{tabular}

\subsubsection{High-rate WPAN mesh}

The high-rate WPAN mesh provides network range extension, reliable communication, and efficient bandwidth reuse in high-rate multimedia applications based on the IEEE 802.15.3 standard [43]. As stated in reference [48], IEEE 802.15.3 defines a protocol for the compatible interconnection of data and multimedia communication equipment via $2.4 \mathrm{GHz}$ radio transmissions in a WPAN. The main purpose of IEEE 802.15.3 is to meet the requirements of portable consumer imaging and multimedia applications by low-power and low-cost systems. In the IEEE 802.15.3 standard, various modulation and coding types are employed in order to support scalable data rates, as shown in Table 1.5. The MAC layer of the standard supports both isochronous and asynchronous data types, and provides the following features [48]:

- ad-hoc peer-to-peer networking;

- fast connections;

- data transport with QoS;

- security.

In order to provide corrections and enhancements to the IEEE 802.15.3 standard, the IEEE 802.15.3b amendment was published in 2005 [49]. IEEE 802.15.3b aims to improve the MAC sublayer by introducing a new definition for the MAC layer management entity (MLME) service access point, and a new acknowledgment policy that allows polling and a more efficient use of channel time. Interested readers are referred to reference [49] for other important additions in IEEE 802.15.3b.

A number of IEEE 802.15.3 devices form a piconet, which is a wireless ad-hoc network that facilitates independent data devices to communicate with each other. One of the devices in a piconet becomes the piconet coordinator (PNC) and provides timing information to the other devices via transmission of beacon signals, as shown in Figure 1.5. In addition, the PNC manages the power-saving modes and the QoS requirements, and controls access to the piconet [48].

The main purpose of the IEEE 802.15.5 standard is to provide an architectural framework to facilitate PNCs in an IEEE 802.15.3 piconet to form a mesh network. In this way, the advantages of mesh networking, listed at the beginning of Section 1.3.2, can be realized. This facilitates various applications, such as coverage extension for multimedia home networking, and improved capacity for interconnection between computers and 


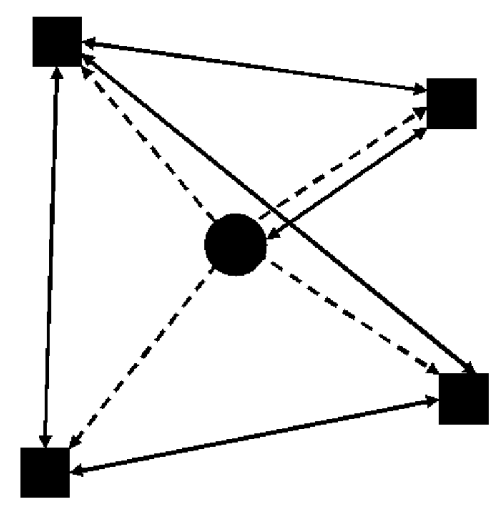

Figure 1.5 Illustration of a piconet, where the circle represents the PNC. The dashed lines indicate beacons sent from the PNC, whereas the solid lines denote data communications.

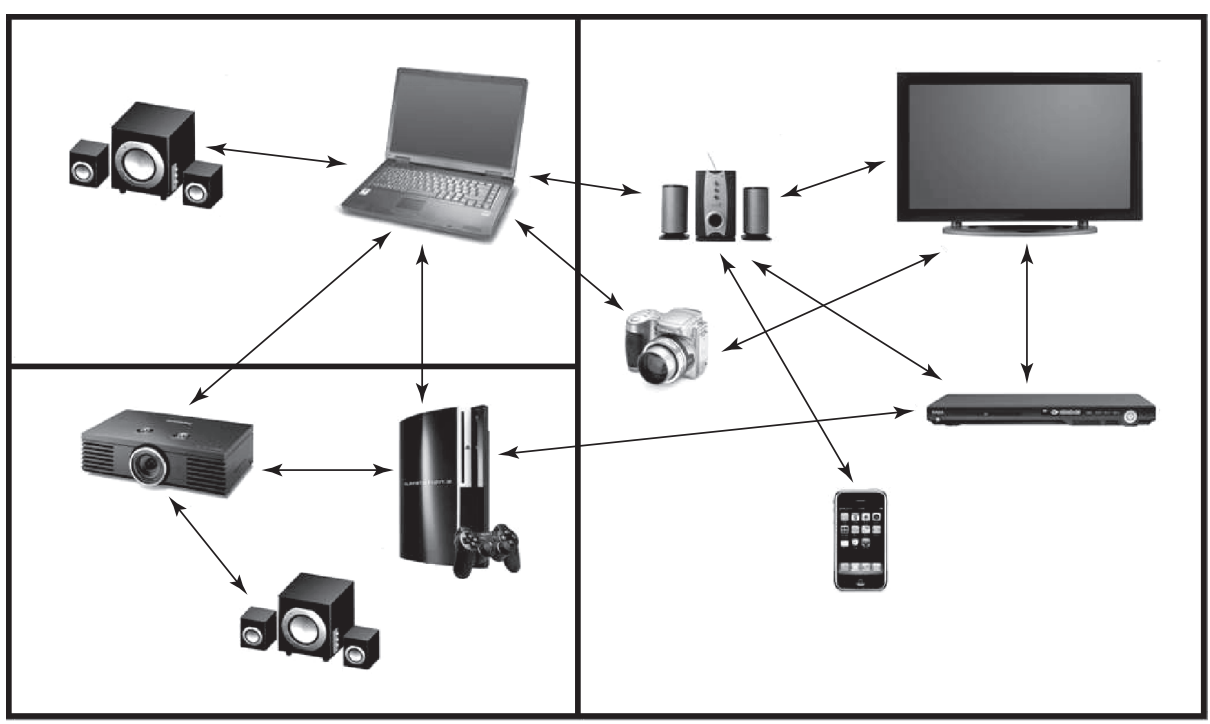

Figure 1.6 Multimedia home networking application of the high-rate WPAN mesh.

peripherals [47]. An example application is illustrated in Figure 1.6, where multimedia networking is implemented in a multiroom house.

\subsubsection{IEEE 802.15 TG6 (body area networks (BANs))}

This ongoing standard is developing a reliable communication technology optimized for low-power devices and operation on, in, or around the human body. Target applications include consumer electronics, medical implants and portable electronics, and personal entertainment. In particular, applications that may benefit from this standard 
include the wireless monitoring of electroencephalogram (EEG), electrocardiogram (ECG), electromyography (EMG), and the monitoring of vital signals. In customizing the technology solution, regulatory issues such as specific absorption rate (SAR) limits are taken into consideration.

The frequency bands supported in the IEEE 802.15.6 standard are 402-405 MHz, 420-450 MHz, 863-870 MHz, 902-928 MHz, 950-956 MHz, 2360-2400 MHz, and 2400-2483.5 MHz. Information data rates provided for these frequency bands are given below.

- 402-405 MHz: $\{57.5,75.9,151.8,303.6,455.4\} \mathrm{Kbps}$.

- 420-450 MHz: $\{57.5,75.9,151.8,187.5\} \mathrm{Kbps}$.

- 863-870 MHz: $\{76.6,101.2,202.4,404.8,607.1\}$ Kbps.

- 902-928 MHz: $\{91.9,121.4,242.9,485.7,728.6\} \mathrm{Kbps}$.

- 950-956 MHz: $\{76.6,101.2,202.4,404.8,607.1\}$ Kbps.

- 2360-2400 MHz: $\{91.9,121.4,242.9,485.7,971.4\}$ Kbps.

- 2400-2483.5 MHz: $\{91.9,121.4,242.9,485.7,971.4\}$ Kbps.

There are several mechanisms to improve communication reliability in BANs including relaying, hybrid ARQ, channel hopping, and interference mitigation.

The BAN supports a star network in which frames are communicated between a coordinator and its end nodes directly. End nodes synchronize their transmissions to a beacon that is periodically transmitted by the body area network (BAN) coordinator. The standard also provides an option for an end node to relay data to the coordinator on behalf of another end node. In this case, an end node is responsible for discovering a secure relay in its range. Overhearing an ACK destined for another end node is used as an indication that the link between the coordinator and that end node is fairly reliable. Therefore, the overhearing node initiates link establishment with the discovered relay end node.

To improve reliability, a hybrid automatic repeat request (HARQ) is adopted. Another reliability improvement comes with channel-hopping. A coordinator may switch to a different channel by including in its beacon the channel-hopping state and the next channel hop fields. It is important that the new channel-hopping sequence is not being used by a different BAN. Switching to a different channel-hopping sequence does not take effect immediately. The coordinator and its end nodes should reside in the current channel for a certain number of beacon periods.

A prospective coordinator can use an interference mitigation mode, in which the coordinator selects a logical channel for network operation while minimizing the impact on the existing BANs. Performing a passive scan on all logical channels in all frequency bands can help the prospective coordinator to estimate other BAN information such as the number of devices on other BANs, their traffic estimates, the data rate used by devices in the other BANs, etc. At the end of the passive scan, the prospective coordinator makes a decision about which logical channel and frequency band to use.

This standard is currently going through a letter ballot and comment resolution. It is expected to be completed in the first half of 2011. 


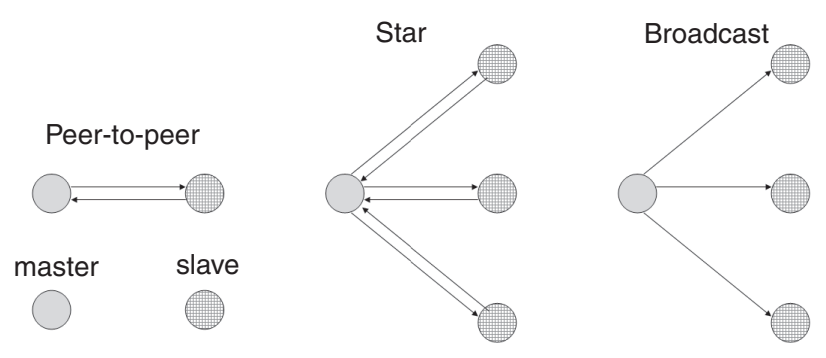

Figure 1.7 Illustration of the three network topologies supported by IEEE 802.15.7 VLC networks: peer-to-peer, star, and broadcast.

\subsubsection{IEEE 802.15 TG7 (visible light communication)}

The IEEE 802.15.7 standard defines both PHY and MAC for short-range optical wireless communications, using visible light in optically transparent media. The visible light spectrum extends from 380 to $780 \mathrm{~nm}$ in wavelength. The standard is required to deliver data rates sufficient to support audio and video multimedia services. Issues such as mobility of the visible link, compatibility with visible light infrastructures, impairments due to noise, and interference from unintended sources such as ambient light are being addressed, because VLC systems may need to coexist with ambient lighting and other optical technologies. Also, the standard is required to abide by any applicable eye safety regulations.

VLC devices are classified as infrastructure, mobile, and vehicle-mounted. The standard supports both uni-directional and bi-directional data, with point-to-point or pointto-multipoint connectivity. The supported topologies are peer-to-peer, star, and broadcast as shown in Figure 1.7.

A PER of $8 \%$ is targeted. The packet size chosen for transmission range evaluation is 256 bytes for low data rate applications and 1024 bytes for high data rate applications.

VLC transmits data by intensity modulating optical sources such as LEDs [50]. Some of the key features of this standard include

- star or peer-to-peer operation;

- optional guaranteed time slots;

- random access with collision avoidance;

- acknowledged transmissions.

The PHY supports three modes:

- Type-I: intended for ranges of tens of meters and low data rate (tens of Kbps) applications. Modulation in this type is ON/OFF keying (OOK) and variable pulse position modulation (VPM).

- Type-II: intended for ranges of tens of meters and moderate data rates in the order of tens of Mbps. This type supports color shift keying (CSK)-based modulation as well as OOK and VPM.

- Type CSK: intended for applications that are using CSK with multiple light sources and detectors. 


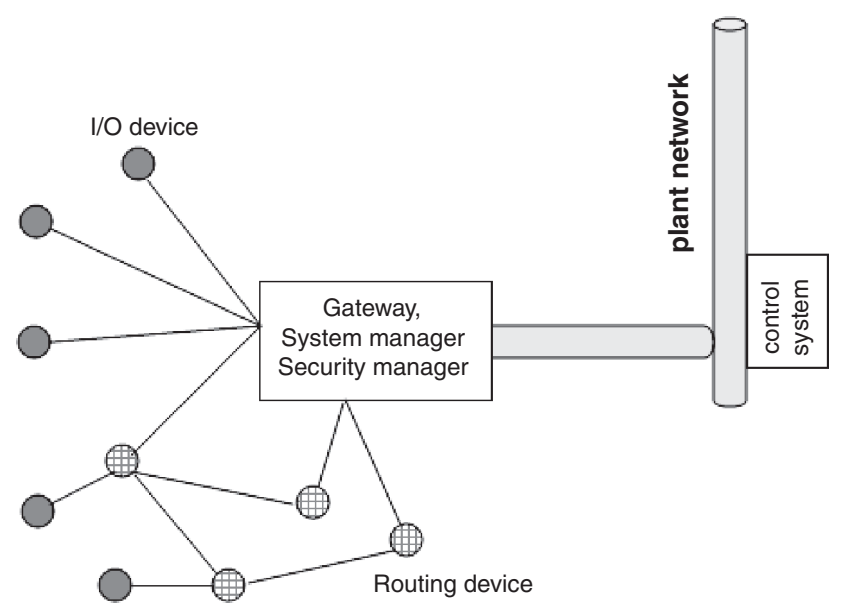

Figure 1.8 Illustration of the SP100.11a supported network topologies for I/O devices and routers.

The standard provides channel hopping to avoid adjacent cell and adjacent coordinator interference. For instance, two coordinators can adopt the following hopping patterns to avoid interference with each other: $\{\mathbf{R}, \mathbf{B}, \mathbf{G}, \mathbf{G}, \mathbf{G}, \mathbf{R}, \mathbf{G}, \mathbf{B}, \mathbf{R}\}$ and $\{\mathbf{G}, \mathbf{G}, \mathbf{R}, \mathbf{B}, \mathbf{R}, \mathbf{G}, \mathbf{B}, \mathbf{R}, \mathbf{G}\}$, where $\mathbf{R}, \mathbf{G}$, and $\mathbf{B}$ denote the red, green, and blue colors, respectively.

This standard is also currently going through a letter ballot and comment resolution. It is expected to be completed in the first half of 2011.

\subsubsection{ISA SP100.11a (process control and monitoring)}

Target applications of SP100.11a are periodic monitoring and process control, where tolerable latency is less than $100 \mathrm{~ms}$. The standard supports simple star topology for portable and $\mathrm{I} / \mathrm{O}$ devices, mesh topology for routing devices, and a combination of the two, as illustrated in Figure 1.8. Path diversity is envisioned to improve reliability.

SP100.11a uses the $2.4 \mathrm{GHz}$ option of the IEEE 802.15.4-2006 as the default PHY. At least 15 frequency channels are supported. A raw PHY data rate is $250 \mathrm{Kbps}$ per channel. Unlike the IEEE 802.15.4 MAC, carrier sense multiple access (CSMA)-based medium access control is made optional, because it may delay transmission of a packet due to random backoff. Instead, the superframe structure is divided into time slots the same as in the time slotted channel hopping (TSCH) configuration of the IEEE 802.15.4e. Each time slot is dedicated to communication between a particular source and destination pair over a prespecified frequency channel hopping pattern. This is referred to as slot hopping in the standard. Channel hopping can be also on a superframe basis. Then, it is called slow hopping. A combination of slot hopping and slow hopping (see Figure 1.9) is also supported.

The ISA SP100.11a standardization was completed in 2009 as being the first industrial wireless networking standard in the ISA100 family of standards. This wireless mesh 


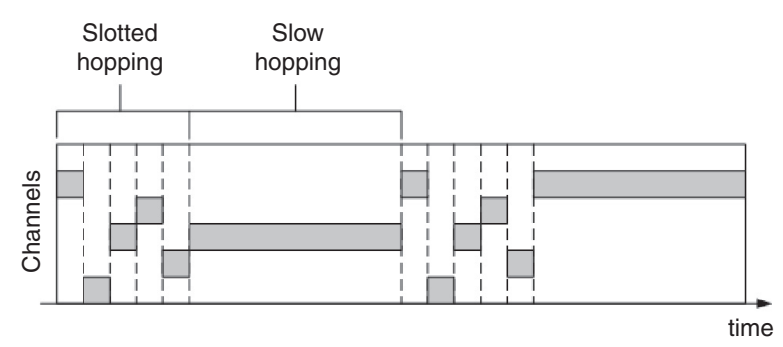

Figure 1.9 Illustration of the hybrid channel hopping operation in SP100.11a, where a number of timeslots using slotted hopping is followed by a slow-hopping period.

network standard protocol helps supplier companies to build inter-operable wireless automation control products.

\section{References}

[1] R. Kraemer and M. D. Katz, Short-Range Wireless Communications: Emerging Technologies and Applications, 1st ed. West Sussex, United Kingdom: John Wiley \& Sons, 2009.

[2] F. F. Dai, Y. Shi, J. Yan, and X. Hu, "MIMO RFIC transceiver designs for WLAN applications," in Proc. IEEE International Conference on ASIC, Oct. 2007, pp. 348-351.

[3] C. C. Lin, S. S. Hsu, C. Y. Hsu, and H. R. Chuang, "A 60-GHz millimeter-wave CMOS RFICon-chip triangular monopole antenna for WPAN applications," in Proc. IEEE Antennas and Propag. Soc. Int. Symp., June 2007, pp. 2522-2525.

[4] P. J. Guo and H. R. Chuang, "A 60-GHz millimeter-wave CMOS RFIC-on-chip meander-line planar inverted-F antenna for WPAN applications," in Proc. IEEE Antennas and Propag. Soc. Int. Symp., July 2008, pp. 1-4.

[5] A. Harney and C. O. Mahony, "Wireless short-range devices: Designing global license-free system for frequencies < 1 GHz," Analog Dialogue Mag., vol. 40, no. 3, pp. 18-22, Mar. 2006.

[6] ECMA International, "High rate ultra wideband PHY and MAC," ECMA-368 Standard, Dec. 2008. [Online]. Available: http://www.ecma-international.org/publications/files/ECMA-ST/ ECMA-368.pdf

[7] — "High rate $60 \mathrm{GHz}$ PHY, MAC, and HDMI PAL," ECMA-387 Standard, Dec. 2008. [Online]. Available: http://www.ecma-international.org/publications/files/ECMA-ST/ ECMA-387.pdf

[8] H. G. Myung, J. Lim, and D. J. Goodman, "Single carrier FDMA for uplink wireless transmission," IEEE Veh. Technol. Mag., vol. 1, no. 3, pp. 30-38, Sep. 2006.

[9] G. Fettweis, E. Zimmermann, V. Jungnickel, and E. Jorswieck, "Challenges in future short range wireless systems," IEEE Veh. Technol. Mag., vol. 1, no. 2, pp. 24-31, June 2006.

[10] W. P. Siriwongpairat, W. Su, M. Olfat, and K. J. R. Liu, "Multiband-OFDM MIMO coding framework for UWB communication systems," IEEE Trans. Sig. Processing, vol. 54, no. 1, pp. 214-224, Jan. 2006.

[11] H. Yang, P. F. M. Smulders, and M. H. A. J. Herben, "Channel characteristics and transmission performance for various channel configurations at $60 \mathrm{GHz}$," EURASIP J. Wireless Commun. Networking, pp. 1-15, Jan. 2007, article ID: 19613. 
[12] A. M. Kuzminsky and H. R. Karimi, "Multiple-antenna interference cancellation for WLAN with MAC interference avoidance in open access networks," EURASIP J. Wireless Commun. Networking, pp. 1-11, Sep. 2007, article ID: 51358.

[13] B. W. Koo, M. S. Baek, Y. H. You, and H. K. Song, "High-speed MB-OFDM system with multiple antennas for multimedia communication and home network," IEEE Trans. Consumer Electronics, vol. 52, no. 3, pp. 844-849, Aug. 2006.

[14] S. M. Mishra, R. W. Brodersen, S. T. Brink, and R. Mahadevappa, "Detect and avoid: An ultrawideband/WiMAX coexistence mechanism," IEEE Commun. Mag., vol. 45, no. 6, pp. 68-75, June 2007.

[15] H. Zhang, X. Zhou, K. Y. Yazdandoost, and I. Chlamtac, "Multiple signal waveforms adaptation in cognitive ultrawideband radio evolution," IEEE J. Select. Areas in Commun., vol. 24, no. 4, pp. 878-884, Apr. 2006.

[16] O. Bakr, M. Johnson, R. Mudumbai, and K. Ramchandran, "Multi-antenna interference cancellation techniques for cognitive radio applications," in Proc. IEEE Wireless Commun. Networking Conf. (WCNC), Budapest, Hungary, Apr. 2009, pp. 1-6.

[17] J. Misic and V. B. Misic, "Performance of cooperative sensing at the MAC level: Error minimization through differential sensing," IEEE Trans. Veh. Technol., vol. 58, no. 5, pp. 24572470, June 2009.

[18] A. Bensky, Short-Range Wireless Communication: Fundamentals of RF System Design and Application, 2nd ed. Elsevier, 2003.

[19] F. H. P. Fitzek and M. D. Katz, Short-Range Wireless Communications - Emerging Technologies and Applications, 1st ed. West Sussex, UK: John Wiley, 2009, ch. 2, pp. 16-23.

[20] R. Kraemer and M. D. Katz, Short-Range Wireless Communications-Emerging Technologies and Applications, 1st ed. West Sussex, UK: John Wiley, 2009, ch. 1, p. 5.

[21] B. Allen, T. Brown, K. Schwieger, E. Zimmermann, W. Malik, D. Edwards, L. Ouvry, and I. Oppermann, "Ultra wideband: Applications, technology and future perspectives," in Proc. Int. Workshop on Convergent Technol. (IWCT), Oulu, Finland, June 2005.

[22] H. Singh, S. K. Yong, J. Oh, and C. Ngo, "Principles of IEEE 802.15.3c: Multi-gigabit millimeter-wave wireless PAN," in Proc. IEEE Int. Conf. Computer Commun. Networks (ICCCN), San Francisco, CA, Aug. 2009, pp. 1-6.

[23] S. K. Yong and C. C. Chong, "An overview of multigigabit wireless through millimeter wave technology: Potentials and technical challenges," EURASIP J. Wireless Commun. Networking, pp. 1-10, Jan. 2007, article ID: 78907.

[24] C. D. Encyclopedia, "ISM band," The Computer Language Company Inc., Jul. 2009. [Online]. Available: http://encyclopedia2.thefreedictionary.com/ISM+band

[25] FCC, "Part 15 - radio frequency devices," ch. I, Title 47 of the Code of Federal Regulations (CFR). [Online]. Available: http://www.access.gpo.gov/nara/cfr/waisidx_05/ 47cfr15_05.html

[26] D. Tse and P. Viswanath, Fundamentals of Wireless Communication. Cambridge, UK: Cambridge University Press, 2005.

[27] J. G. Andrews, A. Ghosh, and R. Muhamed, Fundamentals of WiMAX, 1st ed. Upper Saddle River, NJ: Prentice Hall, 2007.

[28] L. Zheng and D. N. C. Tse, "Diversity and multiplexing: A fundamental tradeoff in multiple-antenna channels," IEEE Trans. Inf. Theory, vol. 49, no. 5, pp. 1073-1096, May 2003.

[29] "IEEE 802.15 Working Group for WPAN." [Online]. Available: http://www.ieee802.org/15 
[30] "Bluetooth SIG." [Online]. Available: http://www.bluetooth.org

[31] IEEE standard for information technology, telecommunications and information exchange between systems, "Local and metropolitan area networks specific requirements, Part 15.2: Coexistence of wireless personal area networks with other wireless devices operating in unlicensed frequency bands," Aug. 2003. [Online]. Available: http://standards.iee.org/ getieee802/download/802.15.2-2003.pdf

[32] “IEEE 802.15 WPAN Task Group 2 (TG2).” [Online]. Available: http://www.ieee802.org/ $15 / \mathrm{pub} / \mathrm{TG} 2 . \mathrm{html}$

[33] “IEEE 802.15 WPAN Task Group 3 (TG3).” [Online]. Available: http://www.ieee802.org/ 15/pub/TG3.html

[34] ECMA-368, "High rate ultra wideband PHY and MAC standard, 1st edition," Dec. 2005. [Online]. Available: http://www.ecma-international.org/publications/files/ECMA-ST/ ECMA-368.pdf

[35] ECMA-369, “MAC-PHY interface for ECMA-368, 1st edition,” Dec. 2005. [Online]. Available: http://www.ecma-international.org/publications/files/ECMA-ST/ECMA-369.pdf

[36] "Ecma International." [Online]. Available: http://www.ecma-international.org

[37] “The International Society of Automation." [Online]. Available: http://www.isa.org

[38] Institute of Electrical and Electronics Engineers, "IEEE Std 802.15.1-2001, wireless medium access control (MAC) and physical layer (PHY) specifications for wireless personal area networks (WPANs)," June 2002. [Online]. Available: http://standards.ieee. org/getieee802/download/802.15.1-2002.pdf

[39] — _ "IEEE Std 802.15.1-2005, wireless medium access control (MAC) and physical layer (PHY) specifications for wireless personal area networks (WPANs), June 2005. [Online]. Available: http://standards.ieee.org/getieee802/download/802.15.1-2005.pdf

[40] D. McCall, "Taking a walk inside Bluetooth EDR," Wireless Net DesignLine, Dec. 2004.

[41] Agilent Technologies, "Bluetooth enhanced data rate (EDR): The wireless evolution," Application Note, May 2006.

[42] "Bluetooth Specification Version 3.0 + HS," Apr. 2009. [Online]. Available: http://www. bluetooth.com/Bluetooth/Technology/Building/Specifications

[43] Institute of Electrical and Electronics Engineers, "IEEE Std 802.15.5-2009, mesh topology capability in wireless personal area networks (WPANs)," May 2009.

[44] "IEEE 802.15 WPAN task group 5 (TG5) mesh networking." [Online]. Available: http:// www.ieee802.org/15/pub/TG5.html

[45] I. F. Akyildiz and X. Wang, "A survey on wireless mesh networks," IEEE Commun. Mag., vol. 43, no. 9, pp. S23-S30, Sep. 2005.

[46] IEEE standard for information technology, telecommunications and information exchange between systems, "Local and metropolitan area networks specific requirements, Part 15.4: Wireless medium access control (MAC) and physical layer (PHY) specifications for low-rate wireless personal area networks (LR-WPANs)," Sep. 2006. [Online]. Available: http://standards.ieee.org/getieee802/download/802.15.4-2006.pdf

[47] M. Lee, "IEEE 802.15.5 WPAN mesh tutorial, IEEE P802.15 working group for wireless personal area networks,” Nov. 2006. [Online]. Available: http://grouper.ieee.org/groups/802/ 802_tutorials/06-November/15-06-0464-00-0005-802-15-5-mesh-tutorial.pdf

[48] IEEE standard for information technology, telecommunications and information exchange between systems, "Local and metropolitan area networks specific requirements, Part 15.3: Wireless medium access control (MAC) and physical layer (PHY) specifications 
for high-rate wireless personal area networks (WPANs)," Sep. 2003. [Online]. Available: http://standards.ieee.org/getieee802/download/802.15.3-2003.pdf

[49] — - "Local and metropolitan area networks specific requirements, Part 15.3: Wireless medium access control (MAC) and physical layer (PHY) specifications for high-rate wireless personal area networks (WPANs: Amendment 1: MAC sublayer)," May 2006. [Online]. Available: http://standards.ieee.org/getieee802/download/802.15.3b-2005.pdf

[50] _ - "Local and metropolitan area networks specific requirements, Part 15.7: Wireless medium access control (MAC) and physical layer (PHY) specifications for Visible Light wireless personal area networks (WPANs: Amendment 1: MAC sublayer)," May 2010. [Online]. Available: http://standards.ieee.org/getieee802/download/d1P802-157-Draft-Standard.pdf

[51] U. C. Kozat, I. Koutsopoulos, and L. Tassiulas, "Cross-layer design for power efficiency and QoS provisioning in multihop wireless networks," IEEE Trans. on Wireless Commun., vol. 5, no. 11, pp. 3306-3315, 2006.

[52] U. C. Kozat, "On the throughput capacity of opportunistic multicasting with erasure codes," in Proc. IEEE 27th Int. Conf. Computer Commun. (IEEE Infocom 2008), Phoenix, AZ, 2008.

[53] J. Chang and L. Tassiulas, "Maximum lifetime routing in wireless sensor networks," in IEEE/ACM Trans. Networking, vol. 12, no. 4, pp. 609-619, 2004.

[54] S. R. Das, C. E. Perkins, E. M. Royer and M K. Marina, "Performance comparison of two on-demand routing protocols for ad hoc networks," IEEE Personal Commun. Mag. special issue on Ad hoc Networking, Feb. 2001, pp. 16-28.

[55] J. Broch, D. A. Maltz, D. B. Johnson, Y. Hu, and J. Jetcheva, "A performance comparison of multihop wireless ad hoc network routing protocols," in Proc. 4th Annual ACM/IEEE Int. Conf. Mobile Computing and Networking (MobiCom'98), Dallas, Texas, October 25-30, 1998.

[56] C. E. Koksal and Hari Balakrishnan, "Quality-aware routing metrics for time-varying wireless mesh networks," in IEEE J. Selected Areas in Commun., vol. 24, pp. 1984-1994, 2006. 